\title{
Simulation of Melt Viscosity Effect on the Rate of Solidification in Polymer
}

\author{
Jaka Fajar Fatriansyah, Hanindito Haidar Satrio, Muhammad Joshua Yuriansyah Barmaki, \\ Arbi Irsyad Fikri, and Mochamad Chalid
}

Department of Metallurgical and Materials Engineering, Faculty of Engineering, Universitas Indonesia, Kampus Depok, West Java 16424, Indonesia

${ }^{*}$ Corresponding author:

email:chalid@metal.ui.ac.id

Received: August 2, 2017

Accepted: August 3, 2018

DOI: $10.22146 /$ ijc. 27195

\begin{abstract}
Phase field model has been successfully derived from ordinary metal phase field equation to simulate the behavior of semi-crystalline polymer solidification phenomenon. To obtain the polymer phase field model, a non-conserved phase field equation can be expanded to include the unique polymer parameters, which do not exist in metals, for example, polymer melt viscosity and diffusion coefficient. In order to expand this model, we include free energy density and non-local free energy density based on HarrowelOxtoby and Ginzburg-Landau theorem for polymers. The expansion principle for a higher order of binary phase field parameter was employed to obtain fully modified phase field equation. To optimize the final properties of the products, the solidification phenomenon in polymers is very important. Here, we use our modified equation to investigate the effect of melt viscosity on the rate of solidification by employing ordinary differential equation numerical methods. It was found that the rate of solidification is related to the melting temperature and the kinetic coefficient.
\end{abstract}

Keywords: polymer; solidification; phase field

\section{- INTRODUCTION}

In manufacturing industries, the use of plastics/polymers as raw materials were steadily increasing due to the suitability of physical properties for many applications in comparison to other materials such as metals. It is reflected from the plastics production rate which grew around $3.4 \%$ in 2015 [1]. The considerations to use the plastics due to their lightweight [2] and relatively inexpensive to be manufactured [3] in comparison with other materials. Facing market demands, some researches have been performed by designing molecular chain of polymers [4], modifying biomass materials into potential polymers [5] and combining conventional polymers with biomass materials [6-7] to answer some challenges in raw materials and processing of polymer products Futhermore, presence of natural fibers in polymer such as polylactic acid and polypropylene strongly implies an effect to crystallization behaviour of the polymers, as reported in literatures [8-9].

In a plastic product manufacturing process, crystallization behaviour of a melted polymer is very important variable in controlling a solidification process to obtain a suitable degree of crystallinity [10]. Furthermore, the plastic crystallinity has a prominent effect to the product properties such as mechanical, thermal, optical and chemical properties.

In order to control the solidification and morphology of melted plastics, the understanding of the solidification mechanism of melted plastics is very important. Plastics solidification mechanism associates the relation between complicated conservation and transport laws as well as micro-phase transformation phenomena governing the plastics/polymers melt flow and solidification. However, in reality, it is very difficult to describe these relations analytically because of the complexity among them. The advances in computational modeling can overcome this predicament, thus enhance the prediction of solidification and morphology during the product manufacturing process.

Solidification of polymers differs from their metals counterparts due to the effect of higher melt viscosity and lower thermal properties, such as melting 
temperature [11-12]. The high melt viscosity, low melting temperature, and partial crystallization feature become the major role to the effect on nucleation growth solidification and morphological evolution in a polymers product manufacturing process. Furthermore, compared with metals counterparts, physical description about solidification kinetics of polymers is more complicated due to envolving a folding behaviour of very long molecular chains.

The solidification of polymers had been modeled using various techniques. Lovinger et al. [13] reported a single crystal growth from the Poly(trifluoroethylene) in the melted phase through a diffusion mechanism approximation. Micheletti et al. [14] investigated the nonisothermal solidification of polymers using a stochastic algorithm which is based on birthand-growth. Raabe et al. [15] examined the kinetics and topology of polymers solidification using three dimensions cellular automaton model. Xu et al. [16] studied isotactic polystyrene single crystals solidification using the phase field method. Within this paper, we modify the phase field model which has been settled in metals counterparts and calculate the solidification rate in polystyrene and other polymers through a simplifying of the phase field model.

Phase-field theory has been used in various problems involving the microstructural evolution of materials. Phase-field theory has been successfully applied to calculate the motion of interfaces and phase boundaries without explicitly tracking those interfaces [17]. For example, this theory has been successful to describe solidification in pure materials/compounds [18] and alloys [19]. Warren et al. [17] and Zhu et al. [20] have developed a two-dimensional phase field model of grain boundary statics and dynamics for polycrystalline materials and polymer modified bitumen, respectively. In this paper, we extend the phase field model and simulate the solidification of the semicrystalline polymer under the effect of melt viscosity using phase field theory.

\section{- EXPERIMENTAL SECTION}

\section{Materials}

In this paper, physical parameters such a melting temperature of polystyrene, polyethylene, polypropylene,
Table 1. The physical parameter of polymers [16,21-22]

\begin{tabular}{lccc}
\hline Polymer & $\mathrm{Tm}_{0}\left({ }^{\circ} \mathrm{C}\right)$ & $\mathrm{Tm}\left({ }^{\circ} \mathrm{C}\right)$ & $\mathrm{T}\left({ }^{\circ} \mathrm{C}\right)$ \\
\hline PS & 243 & 229 & 195 \\
PE & 141 & 133.4 & 120 \\
PC & 288 & 273 & 205 \\
PP & 167 & 158 & 123 \\
\hline
\end{tabular}

and polycarbonate, were used in the simulation (Table 1). All of the polymers can be categorized as thermoplastics due to the existence of both melting point temperature and glass transition temperature as well as their reversibility heating-cooling process.

\section{Theoretical Model}

\section{Landau theory}

Starting with the Ising model of magnetism, the energy of a microscopic system can be described in terms of collections of magnetic spins. This system has a domain which contains atoms where each atom carries a magnetic spin with value $s_{i}= \pm 1$. This value shows whether an atom's magnetic moment is pointing up or down. So, the energy of the system can be written as Eq. (1).

$\mathrm{E}\left\{\mathrm{s}_{\mathrm{i}}\right\}=-\left(\mathrm{J} \sum_{\mathrm{i}}^{\mathrm{N}} \sum_{\mathrm{j}}^{\mathrm{v}} \mathrm{s}_{\mathrm{i}} \mathrm{s}_{\mathrm{j}}+\mathrm{B} \sum_{\mathrm{i}}^{\mathrm{N}} \mathrm{s}_{\mathrm{i}}\right)$

where $J$ and $B$ are the coupling constants or the pure energy that adheres to each spin and its interactions, and the external magnetic field, respectively. The first term of the equation is the sum of the energies due to the interaction of each spin ("i") with all neighboring spins (“j”), while the second term collects the interaction energy of each spin with an externally imposed magnetic field (B). This magnetic field in some cases can also be considered as the chemical potential of the system which can be described as follows

$\mathrm{J}=\beta \mathrm{K}$

$B=\beta h$

$\beta=\frac{1}{\mathrm{k}_{\mathrm{B}} \mathrm{T}}$

where $\mathrm{T}, \mathrm{K}, \mathrm{h}$, and $\mathrm{k}_{\mathrm{B}}$ are temperature, chemical potential, magnetic and Boltzmann constant, respectively.

According to Landau theory, the partition function of the system can be derived from the Ising 
model of magnetism. The canonic partition function of the system $(Z)$ is given by

$\mathrm{Z}=\sum_{\left(\mathrm{s}_{\mathrm{i}}= \pm 1\right)}^{\mathrm{N}} \mathrm{e}^{-\left(\mathrm{J} \sum_{\mathrm{i}}^{\mathrm{N}} \sum_{\mathrm{j}}^{\mathrm{v}} \mathrm{s}_{\mathrm{i}} \mathrm{s}_{\mathrm{j}}+\mathrm{B} \sum_{\mathrm{i}}^{\mathrm{N}} \mathrm{s}_{\mathrm{i}}\right)}$

The average magnetization (" $m$ ") of the system can be considered as the order parameter $\Psi$ which can be easily defined as

$\mathrm{m}=\Psi=\frac{1}{\mathrm{~N}} \sum_{\mathrm{i}}^{\mathrm{N}} \mathrm{s}_{\mathrm{i}}=\left\langle\mathrm{s}_{\mathrm{i}}\right\rangle$

Using Eq. (5), the free energy function (F) can be calculated as

$\mathrm{F}=-\mathrm{k}_{\mathrm{B}} \mathrm{T} \ln (\mathrm{Z})$

$\mathrm{F}=-\mathrm{k}_{\mathrm{B}} \mathrm{T}\left(J \sum_{\mathrm{i}}^{\mathrm{N}} \sum_{\mathrm{j}}^{\mathrm{v}} \mathrm{s}_{\mathrm{i}} \mathrm{s}_{\mathrm{j}}\right)-\mathrm{k}_{\mathrm{B}} \mathrm{T}\left(\mathrm{B} \sum_{\mathrm{i}}^{\mathrm{N}} \mathrm{s}_{\mathrm{i}}\right)$

Using Eq. (8) and the number of spins $\mathrm{N}$, the free energy of each spin $\left(\mathrm{f}_{\mathrm{t}}\right)$ can be expressed as

$f_{t}=\frac{F}{N}=\frac{-k_{B} T\left(J \sum_{i}^{N} \sum_{j}^{v} s_{i} s_{j}\right)}{N}+\frac{-k_{B} T\left(B \sum_{i}^{N} s_{i}\right)}{N}$

From here, we can use the partition function to obtain the free energy per spin by introducing a new term $\mathrm{f}=\frac{-\mathrm{k}_{\mathrm{B}} \mathrm{T}(\mathrm{F})}{\mathrm{N}}$

where $f$ is the free energy function that belongs to the partition function. Substitution of Eq. (10) into Eq. (9) yields Eq. (11).

$\mathrm{f}_{\mathrm{t}}(\mathrm{m}, \mathrm{T})=\frac{\mathrm{F}(\mathrm{m}, \mathrm{T})}{\mathrm{N}}=\mathrm{f}_{\mathrm{i}}+\mathrm{f}_{\mathrm{e}}(\mathrm{m}, \mathrm{T})$

If we consider the variation to be small, then $\mathrm{f}_{e}(\mathrm{~m}, \mathrm{~T})$ can be expanded using Taylor series. However, $\mathrm{f}_{e}(\mathrm{~m}, \mathrm{~T})$ is an even function thus we only need to consider the even terms.

$\mathrm{f}_{\mathrm{e}}(\mathrm{m}, \mathrm{T})=\mathrm{f}_{\mathrm{e}}\left(\mathrm{m}, \mathrm{T}_{0}\right)+\frac{\mathrm{f}_{\mathrm{e}}^{\prime \prime} \mathrm{m}^{2}}{2}+\frac{\mathrm{f}_{\mathrm{e}}^{\prime \prime \prime \prime} \mathrm{m}^{4}}{4}+\ldots$

Put this back into our equation we will obtain

$$
f_{t}(m, T)=\frac{F(m, T)}{N}=f_{i}+f_{e}\left(m, T_{0}\right)+\frac{f_{e}^{\prime \prime} m^{2}}{2}+\frac{f_{e}^{\prime \prime \prime \prime} m^{4}}{4}+\ldots
$$

In this paper, this study is only interested in the perturbation or "noise" that disturb the system. Thus, $\mathrm{f}_{1}+\mathrm{f}_{\mathrm{e}}\left(\mathrm{m}, \mathrm{T}_{0}\right)$ term can be omitted. One thing that needs to be considered is that the first order transitions typically occurs along phases which have different topological symmetry. For instance, there will be broken symmetry occurs. Correspondingly, the first-order transition crosses at a critical point where the two co-existing phases merge into one phase. This symmetry can be solved by adding a cubic order term with a negative sign to the equation.

$$
\begin{aligned}
\mathrm{f}_{\mathrm{t}}(\mathrm{m}, \mathrm{T})= & \frac{\mathrm{F}(\mathrm{m}, \mathrm{T})}{\mathrm{N}}=\mathrm{f}_{\mathrm{i}}+\mathrm{f}_{\mathrm{e}}\left(\mathrm{m}, \mathrm{T}_{0}\right)+\frac{\mathrm{f}_{\mathrm{e}}^{\prime \prime} \mathrm{m}^{2}}{2} \\
& -\frac{\mathrm{f}_{\mathrm{e}}^{\prime \prime \prime} \mathrm{m}^{3}}{3}+\frac{\mathrm{f}_{\mathrm{e}}^{\prime \prime \prime \prime} \mathrm{m}^{4}}{4}
\end{aligned}
$$

\section{Ginzburg-Landau free functional energy}

According to the Ginzburg-Landau theory [23], the interaction energy between substituents can be assumed spatially dependent and varies between any two elements. Let $\nabla$ be the separation between two elements ( $i$ and $j$ ), then the mean internal energy $U$ can be defined as

$\mathrm{U}=\frac{1}{2} \sum_{\mathrm{i}=1 \mathrm{j} \neq \mathrm{i}}^{\mathrm{N}} \epsilon_{\mathrm{ij}}\left(\overrightarrow{\mathrm{x}}_{\mathrm{i}}-\overrightarrow{\mathrm{x}}_{\mathrm{j}}\right) \Psi_{\mathrm{i}}\left(1-\Psi_{\mathrm{j}}\right)$

That equation can be expanded by using the algebraic identity

$\Psi_{i}\left(1-\Psi_{j}\right)=\left(\left[\Psi_{i}-\Psi_{j}\right]^{2}-\left[\Psi_{i}^{2}+\Psi_{j}^{2}\right]+2 \Psi_{i} s\right) / 2$

Assuming that $\mathbb{\nabla}_{\mathrm{ij}}$ is small for any $\mathrm{j}>\mathrm{v}$ and substituting Eq. (16) into Eq. (15), the mean internal energy $(\mathrm{U})$ can be expressed into

$\mathrm{U}=\frac{1}{4} \sum_{\mathrm{i}=1 \mathrm{j} \neq \mathrm{i}}^{\mathrm{N}} \sum_{\mathrm{ij}}\left[\Psi_{\mathrm{i}}-\Psi_{\mathrm{j}}\right]^{2}-\frac{1}{2} \sum_{\mathrm{i}=1}^{\mathrm{N}}\left(1-\Psi_{\mathrm{j}}\right) \Psi_{\mathrm{i}}\left(\sum_{\mathrm{j} \neq \mathrm{i}} \epsilon_{\mathrm{ij}}\right)$

The interaction energy per particle ( can be assumed as $\bigotimes_{\mathrm{ij}} / \nu$, where $\nu$ is the nearest neighbours of elements i. Therefore, the first term of Eq. (17) can be expressed as

$$
\begin{aligned}
\frac{1}{4} \sum_{j \neq 1}\left[\Psi_{i}-\Psi_{j}\right]^{2}= & \frac{a^{2}}{4}\left[\left(\frac{\left[\Psi_{i}-\Psi_{R}\right]^{2}}{a^{2}}+\frac{\left[\Psi_{i}-\Psi_{T}\right]^{2}}{a^{2}}\right)\right. \\
& \left.+\left(\frac{\left[\Psi_{i}-\Psi_{L}\right]^{2}}{a^{2}}+\frac{\left[\Psi_{i}-\Psi_{B}\right]^{2}}{a^{2}}\right)\right]
\end{aligned}
$$

Hence,

$\frac{1}{4} \sum_{j \neq i}\left[\Psi_{i}-\Psi_{j}\right]^{2} \approx \frac{1}{2} \mathrm{a}^{2}\left|\nabla \Psi\left(\overrightarrow{\mathrm{x}}_{\mathrm{i}}\right)\right|^{2}$ 


\section{Phase field model}

Phase-field model is derived based on phase transformation using crystal order parameter concept. This parameter distinguishes two phases, liquid and solid, in term of $\phi$. $\phi$ is defined as 0 and 1 for liquid and solid, respectively. Solidification process can be described as a continuous change of $\psi$ value from zero to one. The solidification process of polymers can be modeled based on Ginzburg-Landau (GL) theory. GL theory incorporates the change of the free energy on boundary to explain the transformation of phase.

This theory explains about solidification of polymer under the effect of change of temperature and distance from the nuclei. In this theory, one needs to define a coefficient of anisotropy in order to induce solidification. In metal solidification, the anisotropy coefficient $[\varepsilon]$ will be greater than zero. However, in polymer solidification, the anisotropy coefficient $[\varepsilon]$ is zero. When the value is greater than zero, the microstructure of the material becomes dendritic. While in the case of zero value, then the microstructure becomes spherulite. In this section, phase field theory is derived from the definition of free energy.

Started from the definition of free energy, classical phase-field model of solidification of virgin material/ compound has free energy that can be written as follows:

$$
\begin{aligned}
\mathrm{F}(\phi) & =\int \mathrm{f}_{\text {local }}(\phi, \mathrm{T}) \mathrm{dV} \\
& =\int \mathrm{f}_{\text {local }}(\phi, \mathrm{T})+\nabla \phi \mathrm{dV}
\end{aligned}
$$

Free energy of the system consists of local free energy $f_{\text {local }}(\phi, T)$ and non-local free energy $f_{\text {grad }}(\phi, T)$. This local free energy $\mathrm{f}_{\text {grad }}(\phi, \mathrm{T})$ can be represented in a gradient term. $\phi$ can be interpreted by phase, but here let use generally interpret $\phi$ as crystal order parameter. The evolution of crystal order parameter can be described as a standard GL approach:

$$
\frac{\partial \phi(\mathrm{r}, \mathrm{t})}{\partial \mathrm{t}}=-\Gamma \frac{\delta \mathrm{F}(\phi)}{\delta \phi(\mathrm{r}, \mathrm{t})}
$$

where $\Gamma$ is the mobility coefficient. This coefficient is defined as inversely proportional to the melt viscosity. To accommodate the dynamics of interfacial surface, the mobility is introduced as a function of the order parameter $\Gamma(\phi)$. In the case of the dynamic of the crystal (solid)-liquid interface, the functional form of $\Gamma(\phi)$ would be more reasonable. Harrowel et al. [24] derived the one dimensional model of the crystal-liquid interface where the time and space evolution of phase $\phi$ can be written as follows:

$\nabla \frac{\mathrm{d} \phi}{\mathrm{dt}}=-\Gamma(\phi) \nabla\left(\frac{\mathrm{df}}{\mathrm{d} \phi}-\kappa \frac{\mathrm{d}^{2} \phi}{\mathrm{dx}^{2}}\right)$

where $\kappa$ is the phase field constant. If the coupling of the motions between different times scales is weak, then the interface between two parameters $\phi_{1}$ and $\phi_{2}$ can be written as:

$$
\begin{aligned}
& \frac{\mathrm{d}^{2} \phi}{\mathrm{dx}^{2}}+\frac{\mathrm{v}}{\Gamma_{1}} \frac{\mathrm{d} \phi_{1}}{\mathrm{dx}}-\frac{\mathrm{d}}{\mathrm{d} \phi_{1}}\left[\mathrm{f}_{0}\left(\phi_{1}\right)+\lambda \mathrm{f}_{1}\left(\phi_{1}, \phi_{1}\right)\right]=0 \\
& \lambda \frac{\mathrm{d}^{2} \phi}{\mathrm{dx}^{2}}+\frac{\mathrm{v}}{\Gamma_{2}} \frac{\mathrm{d} \phi_{2}}{\mathrm{dx}}-\lambda \frac{\mathrm{d}}{\mathrm{d} \phi_{2}} \mathrm{f}_{1}\left(\phi_{1}, \phi_{1}\right)=0
\end{aligned}
$$

The simplest form of $f_{1}$ and related $f_{0}$ can be written as

$\mathrm{f}_{0}\left(\phi_{1}\right)=\mathrm{a}_{1} \phi_{1}{ }^{2}-\mathrm{a}_{2} \phi_{1}{ }^{3}+\phi_{1}^{4}$

Considering polymer within this study, Eq. (25) representing the functional form of free energy, can be transformed into

$\mathrm{f}_{\text {local }}(\phi, \mathrm{T})=\mathrm{W}\left(\frac{\zeta \zeta_{0}}{2} \phi^{2}-\frac{\zeta+\zeta_{0}}{3} \phi^{3}+\frac{\phi^{4}}{4}\right)$

where $\mathrm{W}$ is the height of the energy barrier of nucleation, which can be expressed as

$$
\mathrm{W}=6 \frac{\Delta \mathrm{H}_{\mathrm{u}}}{\mathrm{nRT} \zeta_{0}^{3}}\left(\frac{\mathrm{T}_{\mathrm{m}}-\mathrm{T}}{\mathrm{T}_{\mathrm{m}}^{0}}\right)\left(\frac{\zeta_{0}}{2}-\zeta\right)^{-1}
$$

Parameter $\zeta$ and $\zeta_{0}$ is the unstable energy barrier and the stable solidification potential, respectively. According to $\mathrm{Xu}$ et al. [16], substitution of Eq. (2) and (3) into Eq. (1) obtains Eq (28), expressed as

$$
\frac{\partial \psi(\mathrm{r}, \mathrm{t})}{\partial \mathrm{t}}=-\Gamma\left(\mathrm{W} \psi(\psi-\zeta)\left(\psi-\zeta_{0}\right)-\kappa^{2} \nabla^{2} \psi\right)
$$

Assuming the above equation in one dimension and is observed from a moving frame reference with a uniform velocity of $v=\partial \psi / \partial t$ the Eq. (28) can be rewritten as

$$
\kappa^{2} \frac{\mathrm{d}^{2} \psi}{\mathrm{dx}^{2}}+\frac{v \mathrm{~d} \psi}{\Gamma \mathrm{dx}}+\frac{\partial \mathrm{f}}{\partial \psi}=0
$$


According to literature [24] by setting the boundary condition of $\psi \rightarrow \zeta_{0}$ as $\mathrm{x} \rightarrow-\infty$ and $\psi \rightarrow 0$ as $\mathrm{x} \rightarrow+\infty$, we obtain

$$
\psi(z)=\frac{\zeta_{0}}{1+\exp \left(z \zeta_{0} \sqrt{\frac{\mathrm{W}}{2 \kappa^{2}}}\right)}
$$

In Eq. (29), $\psi$ is a function of $\mathrm{z}$, where $\mathrm{z}=\mathrm{x}$-vt. $\mathrm{v}$ is selected velocity being.

$$
\mathrm{v}=-\Gamma \kappa\left(\zeta-\frac{\zeta_{0}}{2}\right) \sqrt{\mathrm{W}}
$$

Note that this selected velocity does not necessarily represent the growth rate of polymer spherulite. The crystallization kinetics are numerically calculated from Eq. (28) and semi-analytical solution using Eq. (31).

\section{- RESULTS AND DISCUSSION}

The results of the calculation are presented in Fig. 1 and 2. Fig. 1 shows the change of solidification surface in respect of time while Fig. 2 shows the solidification rate in respect of mobility coefficient $\Gamma$. The polymers used are isotactic polystyrene (PS), medium-high density polyethylene (PE), polycarbonate (PC) and polypropylene (PP). All parameters such as melting temperature, melting viscosity density, free energy, kinetic coefficient were obtained from literatures [16,21-22]. According to literature [16], these parameters directly or indirectly

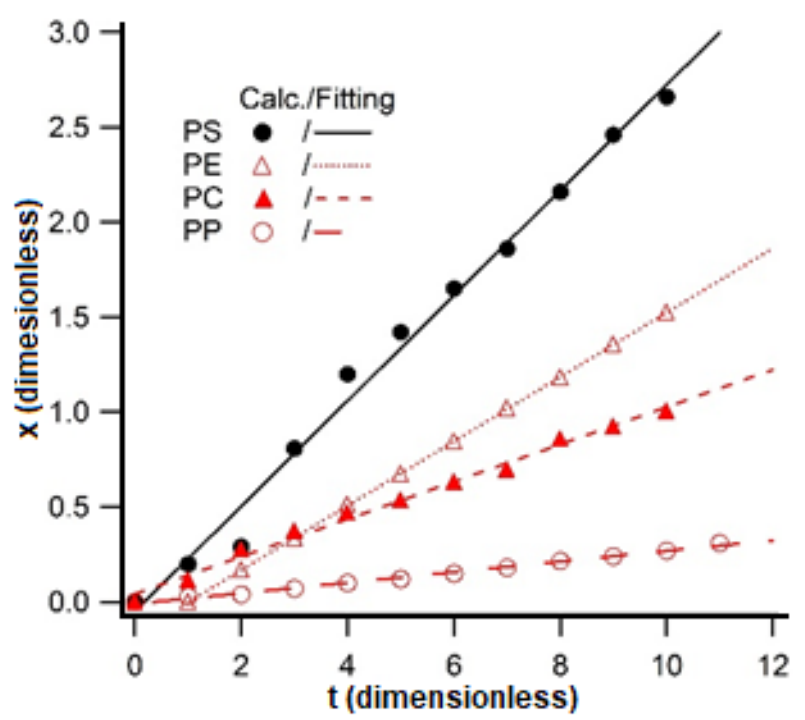

Fig 1. The change of solidification surface in respect of time for PS, PE, PC, and PP related to supercooling, thus leading to various crystal morphologies. So, with the inclusion of supercooling parameter, in the case even our model only captured coarse-grained system results (the change of solidification surface and the solidification rate), it is expected that the results should represent the real phenomena. The other materials (PP, PE, and PC) thermodynamic variables were obtained by rescaling and comparing PS to other materials parameters in respect to the known parameters. Because these stages can not obtain an exact value of the solidification rate, this study only qualitatively compare the solidification rate for each polymer. Obtaining the unexact value of the solidification rate may be caused by complex behaviors of very long molecules during polymer solidification.

Fig. 1 shows the change of solidification surface in respect of time. Dots are data acquired from simulation and lines are fitting curve assuming that the change of solidification surface is constant given small t. The gradient of the curve shows the rate of solidification and thus implying that PS has the fastest solidification rate and it is followed by PE, PC, and PP (see Fig. 2).

According to velocity equation (Eq. (29)), the rate of solidification depends not only on the difference between melting temperature and crystallization temperature but also the height of energy barrier of

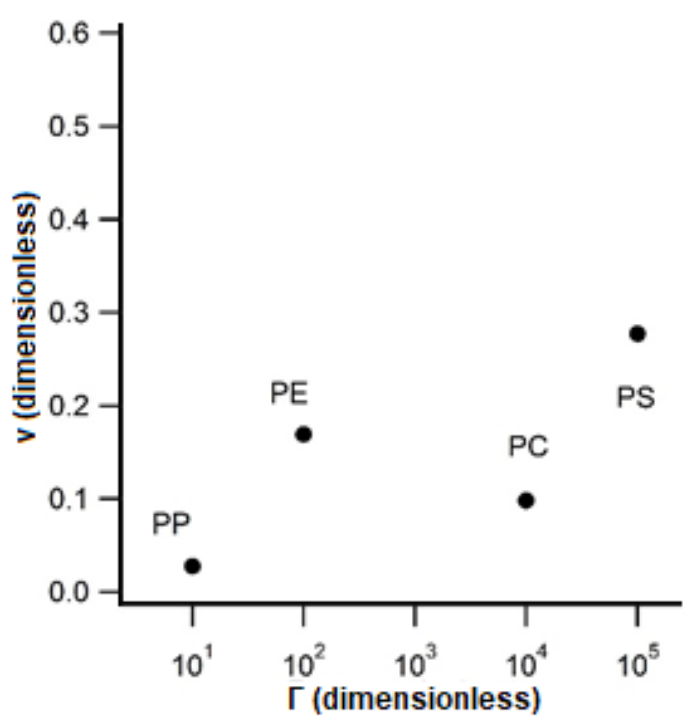

Fig 2. The solidification rate of polymers under the effect of mobility coefficient $\Gamma$ respectively 
nucleation $\mathrm{W}$ and the order parameter $\Gamma(\phi)$. However, as shown by our results (Fig. 1 and 2), the solidification rate is affected mainly by the difference between melting temperature and crystallization temperature. Fig. 2 implies that the lower difference between melting temperature and crystallization temperature, the faster crystallization rate as demonstrated by the solidification rate of all polymers except $P P$.

The exception of PP in Fig. 2, may be caused by neglecting the order parameter in comparing the solidification rate of the polymers. Therefore the order parameter (which is related to the melt viscosity) is more significant parameter in determining solidification rate. PP has very high melt viscosity. This high value yields low mobility or the rotational diffusivity of polymer molecules. The low mobility may yield low solidification rate due to the somewhat low heat transfer between isothermal surface. Although the dynamic of solidification is a rather complex phenomenon, our suggestion above is adequate to explain some peculiar results for $\mathrm{PP}$. The order of solidification rate (from high to low) is PP, PS, and PE, while from the calculation, the order of solidification rate PS, PE, and PP. Based on the results above, calculation of the solidification rate without considering melt viscosity value (or mobility coefficient) gives different results, compared with using the melt viscosity data.

Actually, the mobility value can only be approximated using NMR relaxation spectra which is very difficult to obtain. So, instead of using NMR relaxation spectra, we use more opportunistic approximation by using MFI (melt flow index) value in the polymer. We extrapolated the known NMR relaxation spectra (PS with $\Gamma=10^{5}$ ) with the unknown value. The $\Gamma$ values obtained from gradient for each curves in Fig.1, are then used to calculate the solidification rate (v) shown in Fig. 2. This figure describes correlation of polymer solidification rates with their mobility coefficients. As explained above, the higher mobility coefficient generally translates as higher solidification rate (as shown by PS) and lower mobility coefficient yields lower solidification rate (as shown by PP). However, the relation is not proportional, since PC shows an exception where the solidification rate is even lower than PE. This phenomenon may be caused by relatively high melting and crystallization temperature of PC compared with the others.

\section{- CONCLUSION}

According to our calculation, PS has the fastest solidification rate followed by PE, PC, and PP. Although melting and crystallization rate affect the solidification rate, mobility coefficient can affect solidification rate too. Higher mobility coefficient generally translates to higher solidification rate except for PC due to its high melting and crystallization temperature. The solidification rate model and calculation for polymer/ plastics are very important in plastics manufacturing industries. The information of crystallization time is conspicuous in designing the manufacturing process since it can increase productivity and give information about one cycle production process. Overall discussions in this study indicate that this phase field model is feasible to be applied to PP, PE, PC, and PS.

\section{- REFERENCES}

[1] International Organization for Standardization: Plastic Europe, The plastic industry Berlin, https://committee.iso.org/files/live/sites/tc61/files/ The\%20Plastic\%20Industry\%20Berlin\%20Aug\%20 2016\%20-\%20Copy.pdf, accessed on August 20, 2012.

[2] Corinaldesi, V., Donnini, J., and Nardinocchi, A., 2015, Lightweight plasters containing plastic waste for sustainable and energy-efficient building, Constr. Build. Mater., 94, 337-345.

[3] Atzeni, E., Iuliano, L., Minetola, P., and Salmi, A., 2010, Redesign and cost estimation of rapid manufactured plastic parts, Rapid Prototyping J., 16 (5), 308-317.

[4] Firdaus, D.F., Masrudin, Lestari, D.A., Arbi, M.R., and Chalid, M., 2015, Structure and compatibility study of modified polyurethane/ $/ \mathrm{Fe}_{3} \mathrm{O}_{4}$ nanocomposite for shape memory materials, Indones. J. Chem., 15 (2), 130-140

[5] Chalid, M., Heeres, H.J., and Broekhuis, A.A., 2015, Structure-mechanical and thermal properties 
relationship of novel $\gamma$-valerolactone-based polyurethanes, Polym. Plast. Technol. Eng., 54 (3), 234-245

[6] Faruk, O., Bledzki, A.K., and Fink, H-P., 2014, Progress report on natural fiber reinforced composites, Macromol. Mater. Eng., 299 (1), 9-26.

[7] Chalid, M., Rahman, A., Ferdian, R., Nofrijon, and Priyono, B., 2015, On the tensile properties of polylactide (PLA)/Arenga pinnata "ijuk" fibre composite, Macromol. Symp., 353 (1), 108-114.

[8] Chalid, M., Yuanita, E., and Pratama, J., 2015, Study of alkalization to the crystallinity and the thermal behavior of Arenga pinnata "ijuk" fibers-based poly(lactic acid) (PLA) biocomposite, Mater. Sci. Forum, 827, 326-331

[9] Yuanita, E., Pratama, J., and Chalid, M., 2017, Preparation of micro fibrillated cellulose based on Arenga pinnata 'ijuk' fibre for nucleating agent of polypropylene: Characterization, optimization and feasibility study, Macromol. Symp., 371 (1), 61-68.

[10] Feng, L., Laderman, B., Sacanna, S., and Chaikin, P., 2015, Re-entrant solidification in polymer-colloid mixtures as a consequence of competing entropic and enthalpic attractions, Nat. Mater., 14 (1), 61-65.

[11] Boutaous, M., Zinet, M., Boyard, N., and Bailleul, J.L., 2016, "Phase Change Kinetics within Process Conditions and Coupling with Heat Transfer" in Heat Transfer in Polymer Composite Materials: Forming Processes, Eds., Boyard, N., John Wiley \& Sons, Inc., 121-155.

[12] Le Goff, R., Poutot, G., Delaunay, D., Fulchiron, R., and Koscher, E., 2005, Study and modeling of heat transfer during the solidification of semi-crystalline polymers, Int. J. Heat Mass Transfer, 48 (25-26), 5417-5430.

[13] Lovinger, A.J., and Cais, R.E., 1984, Structure and morphology of poly(trifluoroethylene), Macromolecules, 17 (10), 1939-1945.

[14] Micheletti, A., and Burger, M., 2001, Stochastic and deterministic simulation of nonisothermal crystallization of polymers, J. Math. Chem., 30 (2), 169-193.

[15] Raabe, D., and Godara, A., 2005, Mesoscale simulation of the kinetics and topology of spherulite growth during crystallization of isotactic polypropylene (iPP) by using a cellular automaton, Modell. Simul. Mater. Sci. Eng., 13 (5), 733-751.

[16] Xu, H., Matkar, R., and Kyu, T., 2005, Phase-field modeling on morphological landscape of isotactic polystyrene single crystal, Phys. Rev. E: Stat. Nonlinear Soft Matter Phys., 72, 011804.

[17] Warren, J.A., Kobayashi, R., Lobkovsky, A.E., and Carter, W.C., 2003, Extending phase field models of solidification to polycrystalline materials, Acta Mater., 51 (20), 6035-6058.

[18] Collins, J.B., and Levine, H., 1985, Diffuse interface model of diffusion-limited crystal growth, Phys. Rev. B: Condens. Matter, 31 (9), 6119-6122.

[19] Wheeler, A.A., Boettinger, W.J., and McFadden, G.B., 1992, Phase-field model for isothermal phase transition in binary alloys, Phys. Rev. A: At. Mol. Opt. Phys., 45 (10), 7424-7439.

[20] Zhu, J., Lu, X., Balieu, R., and Kringos, N., 2016, Modelling and numerical simulation of phase separation in polymer modified bitumen by phase field method, Mater. Des., 107, 322-332.

[21] Morris, P.J., 2005, Polymer Pioneers: A Popular History of the Science and Technology of Large Molecules, Chemical Heritage Foundation, Philadelphia, PA 19106, USA, 76.

[22] Kaiser, W., 2011, Kunststoffchemie für Ingenieure Von der Synthese bis zur Anwendung, $3^{\text {rd }}$ ed., Carl Hanser Verlag GmbH \& Co. KG, München.

[23] Provatas, N., and Elder, K., 2005, "Ising Model of Magnetism" in Phase-Field Methods in Material Science and Engineering, Wiley-VCH, New York, 11-14.

[24] Harrowell, P.R., and Oxtoby, D.W., 1987, On the interaction between order and a moving interface: Dynamical disordering and anisotropic growth rates, J. Chem. Phys., 86, 2932-2942. 